\title{
Crisis de los cuidados y crisis sistémica: la repro- ducción como pilar de la economía llamada real
}

\author{
Sandra EZQUERRA \\ Universitat de Vic (Barcelona) \\ Sandra.Ezquerra@uvic.cat
}

Recibido: 10.12.2011

Aceptado: 20.01.2012

\section{RESUMEN}

En el presente texto se analizan los principales factores de la crisis de los cuidados. Se muestra que el estado y los mercados han evadido abordar las premisas y desigualdades que yacen tras dicha crisis y han intentado mitigarla promoviendo su externalización e internacionalización. Por otro lado, la gestión neoliberal de la crisis sistémica en los últimos años agrava y cronifica la crisis de los cuidados mediante una re-privatización de la reproducción en aras de la supervivencia y enriquecimiento de los mercados. Esto nos recuerda el íntimo vínculo existente entre la economía productiva y la reproductiva, así como la centralidad de los cuidados y la reproducción en lo que muchos se empeñan en seguir llamando economía real.

Palabras clave: crisis de los cuidados, crisis sistémica, reproducción, neoliberalismo, inmigración.

\section{Care giving and structural crisis: reproduction as the keystone of the so-called real economy}

\begin{abstract}
In this text I analize the main factors behind the care giving crisis. I show that both the state and the markets have refused to address the premises and inequalities standing behind such crisis and have tried to mitigate it by promoting its externalization and internationalization. At a different level, the neoliberal management of the structural crisis during the past years worsens and perpetuates the care giving crisis through a re-privatization of reproduction in the aim of supporting the survival and enrichment of markets. This reminds us of the intimate link existing between the productive economy and the reproductive one, as well as of the centrality of care giving and reproduction within what many people insist in calling real economy..
\end{abstract}

Key words: Political propaganda, Female objectification, beautiful symbols.

\section{INTRODUCCIÓN}

Una de las principales aportaciones de la economía feminista en los últimos años ha sido la visibilización de las profundas limitaciones resultantes de la atención exclusiva de la economía neoclásica en los mercados, las actividades monetarizadas y los procesos de acumulación capitalistas. Dicha concentración no sólo presenta un sesgo de género importante dejando fuera del análisis económico a una parte importante de las mujeres del planeta, sino que además analiza la esfera de la economía mal llamada productiva como si fuera independiente respecto a otros 
ámbitos como la familia y los trabajos que en su seno se realizan. Esta especialización de la economía convencional en "lo productivo" ignora la existencia de vínculos profundos y dialécticos entre la economía de mercado y el cuidado de las personas y la reproducción de la vida, relegando estos últimos a la entidad de fenómenos secundarios y no económicos pertenecientes al ámbito del hogar y la intimidad. El hecho de que estas actividades hayan sido realizadas tradicionalmente por las mujeres de forma no remunerada en el marco de la familia contribuye a su invisibilización ${ }^{1}$.

Diversas transformaciones sociales, económicas, políticas y demográficas acontecidas en las últimas décadas, sin embargo, han descubierto la importancia que para la economía tiene lo que sucede en los hogares y han puesto de manifiesto la fragilidad del equilibrio-separación entre ámbito público y privado sobre el que descansa la economía capitalista, provocando así la llamada crisis de los cuidados. Por crisis de los cuidados nos referimos a la puesta en evidencia y agudización de las dificultades de amplios sectores de la población para cuidarse, cuidar o ser cuidados. Dichas dificultades se manifiestan a raíz de una desestabilización del modelo tradicional de reparto de las responsabilidades sobre los cuidados y una reestructuración del conjunto del sistema socioeconómico, sin que se haya alterado por ello la división sexual del trabajo en los hogares ni la segmentación de género en el mercado laboral. Lejos de atañer exclusivamente al ámbito del cuidado y la reproducción, entendemos que la crisis de los cuidados es síntoma y resultado de la profundización de las tensiones-divisiones que el capitalismo patriarcal ocasiona y sobre las que se erige, entre privado y público, entre reproductivo y productivo y, en última instancia, entre la satisfacción de las necesidades humanas y la generación de beneficio económico.

Aunque a priori pueda parecer contrario a la intuición, la aparición de la crisis de los cuidados presenta tanto inconvenientes como potencialidades. El inconveniente más visible es el vacío o ausencia de cuidados resultante de los cambios descritos o la generalización de la dificultad de amplios sectores de la población para poder cuidarse, cuidar o ser cuidados en diferentes momentos de su ciclo vital. $\mathrm{La}$ principal potencialidad de la crisis de los cuidados, por otro lado, yace sin duda en su visibilización de la insostenibilidad de la organización tradicional del cuidado, así como de la naturaleza inherentemente injusta y parasitaria respecto al trabajo reproductivo de la economía llamada productiva. La economía reconocida y valorada como real ha dependido históricamente de un trabajo invisible, gratuito y supuestamente altruista impuesto a las mujeres en la sombra de los hogares y las familias. La relegación social, cultural y física de las mujeres a esta esfera ha resultado imprescindible para garantizar la supervivencia y reproducción del sistema

\footnotetext{
${ }^{1}$ La economía neoclásica no ha estado sola en su exclusión e invisibilización de la esfera reproductiva y la mayoría de corrientes heterodoxas de pensamiento económico, incluyendo el marxismo, han incurrido en el mismo error.
} 
económico. La crisis de los cuidados ofrece una valiosa oportunidad para poner de manifiesto por un lado la importancia del cuidado y la reproducción como motor invisible de la economía y, por el otro, la opresión de género inherente a esta relación parasitaria entre ambas esferas. Dicho de otro modo, ayuda a dilucidar la subordinación de la lógica del bienestar y el cuidado a la lógica del beneficio económico, y muestra de manera clara el profundo vínculo existente entre las relaciones patriarcales y las del capital. Ofrece el potencial, además, de ayudar a repensar las premisas y el orden de prioridades sobre las que se erige el sistema así como de proponer alternativas radicalmente distintas. Dicha oportunidad, sin embargo, no está siendo aprovechada y el riesgo de lo que Amaia Pérez Orozco denomina en este mismo volumen un cierre reaccionario de la crisis de los cuidados o, peor aún, de su cronificación y ahondamiento, es cada vez mayor. Este riesgo se ve agudizado en el actual momento de crisis sistémica.

En el presente texto se analizan, en primer lugar, algunos de los principales factores que han generado la crisis de los cuidados. A continuación se examinan algunas de las respuestas políticas, económicas y sociales a ella y, en tercer lugar, se reflexiona sobre su externalización e internacionalización. Tanto en el segundo como en el tercer apartado se realiza un esfuerzo para demostrar que la actual crisis sistémica ha contribuido al cierre reaccionario, agravamiento y cronificación de la crisis de los cuidados y, en realidad, las respuestas políticas a la segunda se encuentran regidas por los mismos paradigmas desde los que se está abordando la primera. Éstos, en una actualización de los procesos de acumulación originaria como salvaguarda del sistema económico, devuelven una parte importante de las responsabilidades del cuidado y la reproducción previamente asumidas por el estado a las mujeres en los hogares, y su gestión a los mercados, como parte de una estrategia de re-privatización de la reproducción en aras del enriquecimiento de los mercados y la supervivencia de la economía considerada real.

\section{CRISIS DE LOS CUIDADOS: ¿CÓMO HEMOS LLEGADO HASTA AQUí?}

Numerosos países del mundo, entre ellos el Estado español, vienen sufriendo en los últimos años lo que se ha venido a llamar crisis de los cuidados. Por crisis de los cuidados se entiende la puesta en evidencia de la incapacidad social y política de garantizar el bienestar de amplios sectores de la población (Ezquerra, 2010b) y la generalización de la dificultad de éstos para poder cuidarse, cuidar o ser cuidados (Del Río, 2004). Dichas dificultades se manifiestan tras un complejo proceso de desestabilización del modelo tradicional de reparto de responsabilidades sobre los cuidados y la sostenibilidad de la vida y una reestructuración del conjunto del sistema socioeconómico (Pérez Orozco, 2006). Esta desestabilización conlleva una reorganización simultánea del trabajo asalariado y no asalariado, sin alterar no obstante la rígida división sexual del trabajo en los hogares ni la segmentación de género en el mercado de trabajo remunerado (Rico, 2009). 
Los principales factores de la crisis de los cuidados se agrupan en tres categorías distintas: transformaciones socio-demográficas, socio-laborales y político-económicas. Si bien resulta del todo imposible comprender estos fenómenos de manera aislada por encontrarse íntimamente relacionados entre ellos, desde un punto de vista analítico se considera útil examinarlos por separado. En primer lugar destacan el envejecimiento de la población y el aumento de la esperanza de vida, los cuáles han desembocado durante los últimos años en una creciente demanda de cuidado. En segundo lugar hemos presenciado desde los años 80 una importante disminución de la disponibilidad de las mujeres para cuidar en el hogar y, por consiguiente, de la oferta de cuidado. En tercer lugar venimos asistiendo a la expansión de las políticas neoliberales de recortes sociales, las cuales limitan severamente los recursos públicos para cubrir el vacío generado por el aumento de la necesidad de cuidado y el descenso de personas (léase mujeres) disponibles para llevarlo a cabo. Todas estas circunstancias, junto con la ausencia de corresponsabilidad por parte de los mercados y la mayoría de los hombres respecto a las tareas del cuidado, han desembocado en un problema social de primera magnitud (Hernando,2006; Ezquerra, 2010a; Setién \& Acosta, 2010)

Tras la Segunda Guerra Mundial la economía internacional vivió una etapa de gran expansión económica durante la cual se consolidó una clara división sexual del trabajo en el seno de las familias que ubicaba al hombre-marido en el rol de sustentador y a la mujer-esposa en el de ama de casa/cuidadora. Este período de relativa bonanza y estabilidad de los roles de género vio su fin con la recesión de los años 70. Las estrategias del capital para incrementar su competitividad y su tasa de beneficio desde entonces han estado centradas en la generalización de políticas deflacionistas y de contención salarial. La demonización neoliberal de la deuda pública ha tenido a su vez un impacto negativo sobre el gasto público social.

Se ha presenciado a su vez desde entonces un espectacular incremento de la participación de las mujeres en el mercado laboral o lo que se ha denominado la feminización del empleo a escala global, lo cual ha coincidido con su creciente flexibilización. La existencia y disponibilidad de una nueva fuerza de trabajo percibida por el capital como dócil y de bajo coste ha sido una de las piezas claves de la apuesta neoliberal de precarización del empleo en nombre del libre mercado, la competitividad global y la eficiencia económica (véase Marchand \& Runyan, 2000; Ferber \& Nelson, 2003; Salzinger, 2003; Gálvez \& Torres, 2010). Ello ha provocado importantes alteraciones en el "consenso de género" que había caracterizado la era de postguerra en el ámbito de la familia, las cuales no pueden comprenderse de manera independiente a las transformaciones acontecidas en la esfera de los mercados y, particularmente, del laboral. Ambos espacios, así como la relación dialéctica existente entre ellos, son factores fundamentales tras la crisis de los cuidados.

El estado español no ha sido inmune a estos procesos. Tal y como se muestra en el Gráfico 1, desde la década de los 80 la "tasa de actividad" femenina en el merca- 
do de trabajo remunerado se ha incrementado de manera notable. Mientras que en el año 1976 era de 28,67\%, en el tercer trimestre del 2011 se acercaba al 53\%:

Gráfico 1. Tasas de actividad en el mercado de trabajo remunerado por género. 1976- 2011. \%

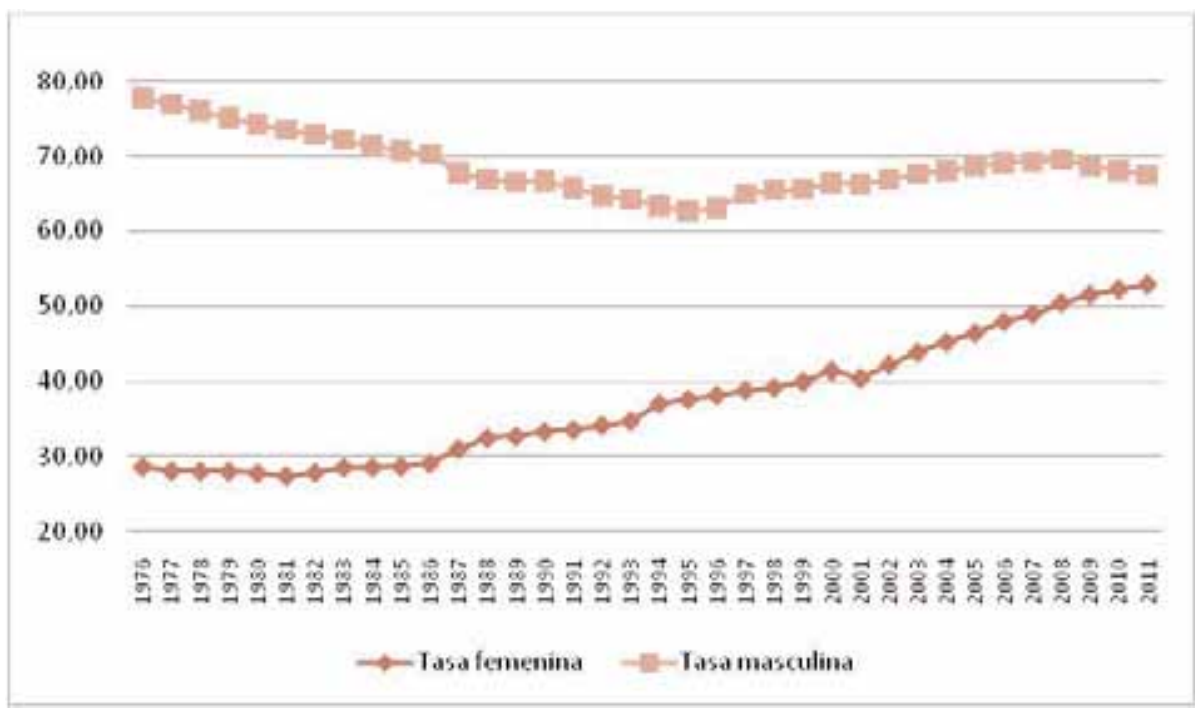

Elaboración propia a partir de los datos de la Encuesta de Población Activa

En las últimas décadas hemos asistido a una creciente participación de las mujeres españolas en el mercado de trabajo remunerado. Dicho crecimiento se ha mantenido desde que estalló la crisis sistémica mientras que la tasa de participación masculina se ha estancado e incluso reducido. El mundo del empleo ha dejado de ser una esfera vetada para la mayoría de las mujeres, y la identificación social de éstas con la arena doméstica y del cuidado ha constituido cada vez menos una razón, coartada o justificación para dicho veto. El Gráfico 2 ilustra la pérdida de fuerza, a la hora de explicar nuestra exclusión del mercado laboral, del rol tradicional que naturaliza a las mujeres como cuidadoras, ya que el número de personas en situación "económicamente inactiva" como resultado de sus responsabilidades en las "labores del hogar" ha disminuido de manera importante: mientras que en 1976 un $65,52 \%$ de las personas contabilizadas como ajenas al mercado laboral atribuían su situación a sus responsabilidades domésticas y de cuidado, en el año 2011 dicho porcentaje se había reducido a menos de la mitad hasta llegar al 27,71\%. 
Gráfico 2. Dedicación a las “labores del hogar” como causa de inactividad en el mercado de trabajo remunerado. 1976-2011, \%

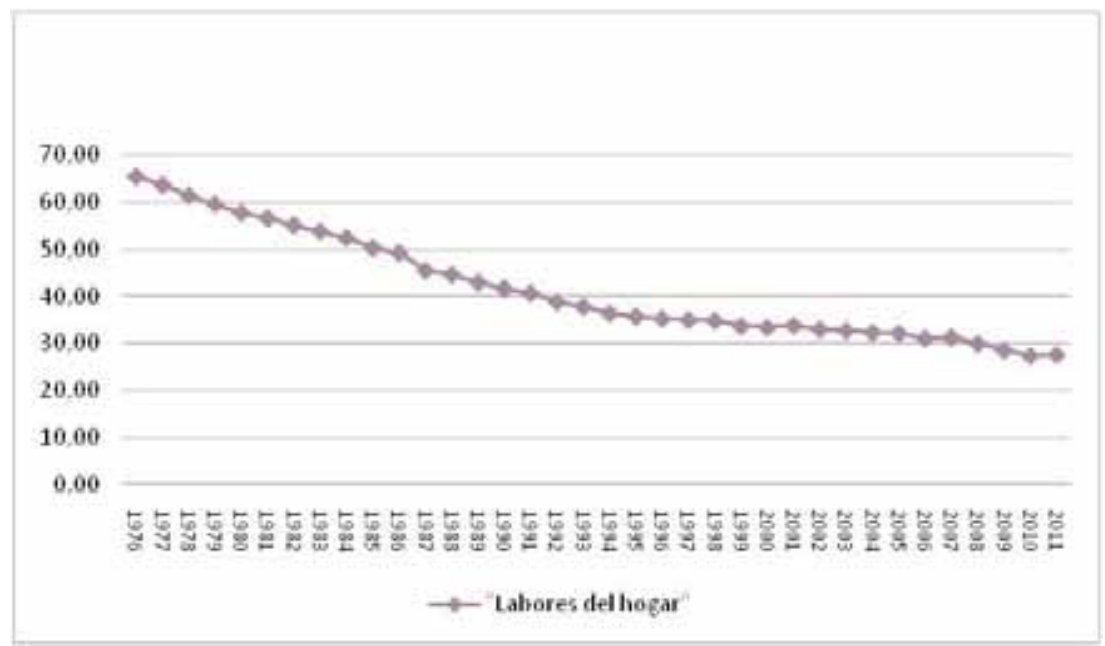

Elaboración propia a partir de los datos de la Encuesta de Población Activa

Si se tiene en cuenta que, tal y como los datos del Gráfico 3 muestran de manera flagrante, durante las cuatro últimas décadas las mujeres han constituido entre el $91 \%$ y el $100 \%$ de las personas cuya ausencia del mercado de trabajo remunerado respondía a responsabilidades domésticas y de cuidado, podemos afirmar con seguridad que durante todo este tiempo una parte importante de la población femenina ha dejado de ver su rol de esposa, madre o hija cuidadora como un impedimento absoluto a su incorporación al mundo del empleo.

Gráfico 3. Personas inactivas en el mercado laboral remunerado por dedicarse a las "labores del hogar” por género. 1976-2011, \%

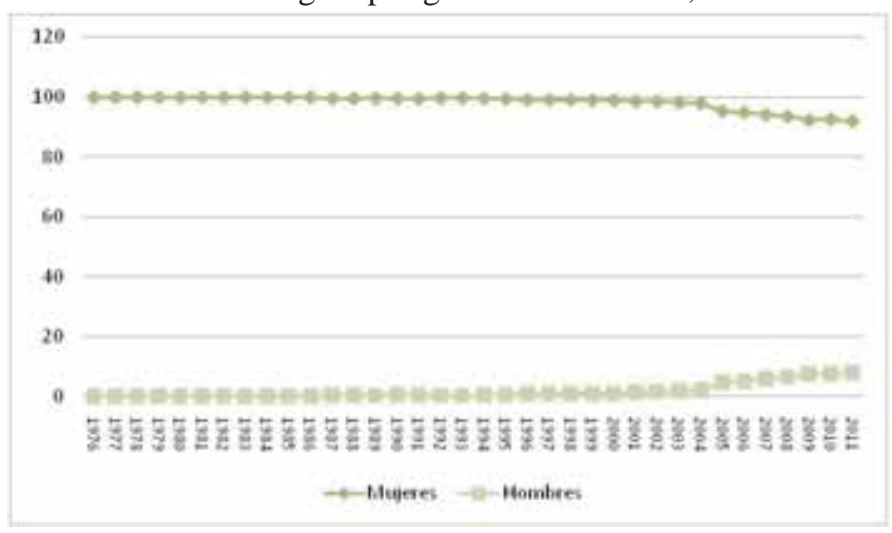

Elaboración propia a partir de los datos de la Encuesta de Población Activa 
Sin embargo, la pérdida de peso de las responsabilidades domésticas y del cuidado a la hora de explicar la ausencia de las mujeres del mercado de trabajo remunerado ni ha cubierto las necesidades sociales reproductivas ni las ha hecho desaparecer por arte de magia. En realidad éstas han aumentado: la creciente presencia de las mujeres en el mercado laboral se ha dado de manera paralela a un envejecimiento de la población y un incremento de la esperanza de vida. Así, tal y como se refleja en el Gráfico 4, mientras que miles de mujeres han abandonado durante las últimas décadas su especialización en el cuidado, el número de personas que o bien por su elevada edad o bien por su diversidad funcional necesitan atención, ha aumentado. La creciente divergencia entre las cifras correspondientes a la oferta de cuidadoras y la demanda de cuidado plasma visualmente a la perfección el concepto y realidad de la crisis de los cuidados:

Gráfico 4. Mujeres “inactivas” dedicadas a las “labores del hogar” y personas con necesidad de atención, 1996-2011, unidades

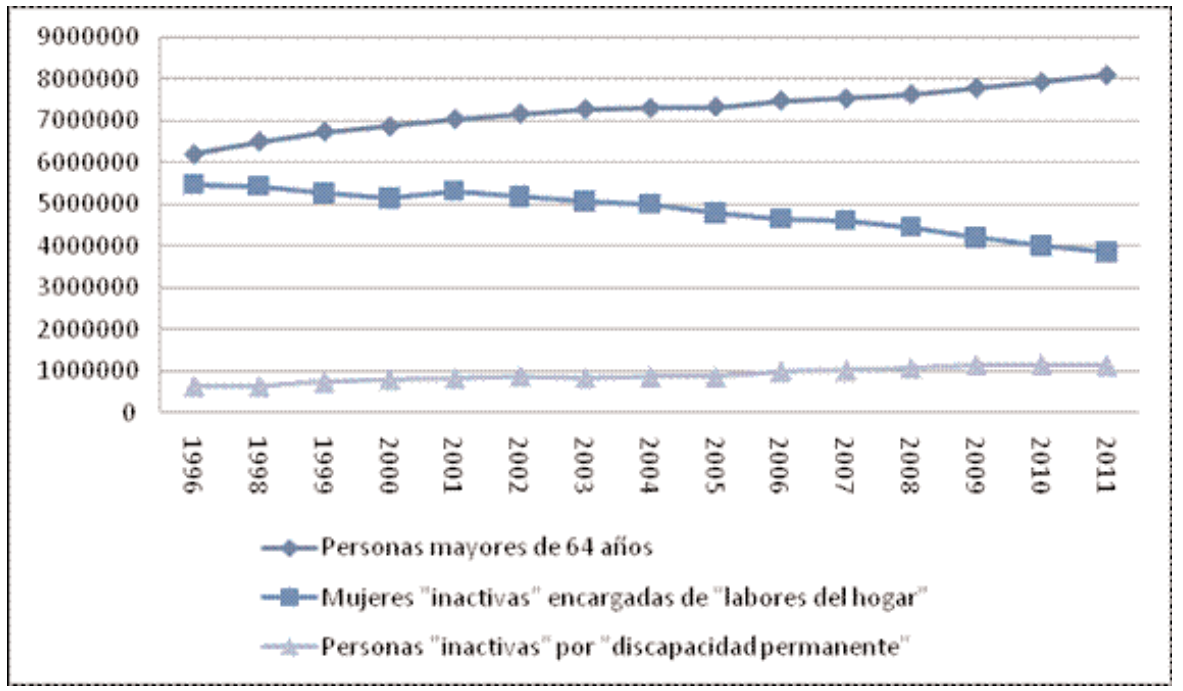

Elaboración y cálculos propios a partir de los datos del Instituto Nacional de Estadística y de la Encuesta de Población Activa

Ante estos cambios cabe realizar dos reflexiones. En primer lugar, a pesar de los avances recientes, a inicios de la segunda década del siglo XXI todavía hay en el estado español 2.235.300 de mujeres que no buscan un empleo remunerado porque o bien tienen que cuidar de niños o personas adultas en situación de autonomía restringida o bien tienen otras responsabilidades familiares o personales ${ }^{2}$. La cifra

\footnotetext{
2 Somos consciente de que un número importante de estas mujeres seguramente tienen un empleo en la economía informal. Es difícil, no obstante, establecer cuántas y, en todo caso, se encuentran excluidas del mercado laboral formal con la pérdida de derechos sociales y laborales que ello comporta.
} 
de hombres en la misma situación es de 138.500 y constituye apenas un 6\% de la de mujeres. Dicho de otro modo, un número importante de mujeres continúa considerando que sus responsabilidades domésticas y de cuidado no son compatibles con su participación en el mercado laboral.

En segundo lugar, en relación a las que sí se incorporan al mundo del trabajo remunerado, es necesario interrogarse sobre la manera en que la feminización del empleo afecta a la organización tradicional del cuidado. Dicho de otro modo, una vez que la incorporación generalizada de las mujeres en el mercado laboral empieza a resquebrajar el precario equilibrio que garantizaba el cuidado de las personas y la reproducción de la vida, y teniendo en cuenta que estas necesidades no disminuyen sino que aumentan, ¿cuáles son las consecuencias? ¿Quién asume la responsabilidad? ¿Y qué efectos tienen estos cambios en las vidas de las mujeres y las familias?

\section{RESPUESTAS A LA CRISIS DE LOS CUIDADOS: ESTADOS, MERCA- DOS Y HOMBRES}

A continuación se exponen los rasgos generales de las respuestas a la crisis de los cuidados en el estado español provenientes de tres ámbitos políticos, económicos y sociales respectivamente: el estado, los mercados y los hombres.

En lo que se refiere al estado, se analiza su postura durante los últimos años respecto al cuidado de niños y niñas por un lado y de personas en situación de autonomía restringida o con diversidad funcional por el otro. Se examina a su vez hasta qué punto dicha postura se ha visto complementada y/o socavada por las respuestas políticas a la crisis sistémica también fuera del ámbito de los cuidados.

Mientras que en años recientes hemos presenciado un cierto aumento de escuelas infantiles públicas, en la actualidad únicamente el $10 \%$ de niños y niñas de 0 a 2 años del estado español tienen acceso a plaza en comparación con el $28 \%$ de la UE-15 o el 58\% de Suecia. A su vez, en 2004 sólo un 2\% de las personas en situación de autonomía restringida tenían atención garantizada frente al 18\% de la UE15 y el 23\% de Suecia (Navarro, Torres \& Garzón, 2011: 117). A principios de noviembre del 2011, de las 2.142.004 millones de personas que habían solicitado la prestación desde la entrada en vigor de la Ley de Dependencia en 2007, únicamente 1.207.773 (56,4\%) eran beneficiarias de la prestación (SAAD-IMSERSO, 2011). Además de lo cuestionable de su supuesta universabilidad y su financiación pública, el texto de la ley ha dejado la puerta abierta para la gestión privada de los servicios, lo cual tiene un impacto negativo tanto en la calidad del servicio de cuidado como en las condiciones laborales de las personas que lo llevan a cabo (Hernando, 2006). Por otro lado, mediante la generalización de las ayudas a personas (léase mujeres) cuidadoras, la ley ha potenciado durante los últimos años la continuidad del confinamiento de las mujeres en el ámbito privado y no ha cuestionado en ningún caso la división sexual del trabajo. 
El autismo de las administraciones respecto a la crisis de los cuidados se está viendo agravado recientemente por su apuesta de gestión neoliberal de la crisis sistémica. El gasto social español per cápita no llega al 70\% de la media de la UE-15. En lo que se refiere a algunos servicios públicos como la atención a la infancia, en lugar de marcarse el objetivo de acortar distancias respecto a las cifras europeas, algunas administraciones están realizando una apuesta por la privatización de la atención a la infancia ${ }^{3}$. Por otro lado, si bien en el Plan Español para el Estímulo de la Economía y el Empleo el año 2008 se mencionaban inversiones sociales y el apoyo a las familias como ejes principales (Gobierno de España, 2008), de los 11.000 millones de euros que se inyectaron durante el primer año de la crisis para crear empleo, la mayoría fueron a parar al sector de la construcción. Únicamente 400 millones fueron destinados a Dependencia y, aún así, un grueso importante de esta cantidad se invirtió en infraestructuras ${ }^{4}$ (Otxoa, 2009).

El siguiente estadio de la crisis, inaugurado formalmente en mayo de 2010 y marcado por la santificación oficial del déficit cero, ha presenciado la supresión del cheque bebé y de la retroactividad de las ayudas a la dependencia 5 . En lo que se refiere a políticas relacionadas con los cuidados, cabe destacar también la renuncia del gobierno del PSOE a ampliar el permiso de paternidad, su supresión del Ministerio de Igualdad o su apoyo al bloqueo europeo a las mejoras en los permisos de maternidad. La profundización de las políticas neoliberales durante los últimos dos años, encarnadas en una desaparición, disminución, privatización y/o pérdida de calidad de los servicios, permisos y prestaciones sociales, están siendo acusadas de forma particularmente grave por las mujeres ya que, ante la desaparición de servicios y ayudas sociales por un lado y la pervivencia de la división sexual del trabajo, somos nosotras las que nos hacemos cargo mediante nuestro trabajo invisible y altruista de cuidados y un incremento importante de nuestra carga total trabajo ${ }^{6}$ (Gálvez \& Torres, 2009; Ezquerra, 2011a).

En lo que se refiere a los mercados, concretamente el mercado laboral, cabe destacar tres dimensiones distintas: primero, las condiciones laborales en las que

3 Uno de los casos que ha recibido más atención social y mediática en los últimos meses ha sido el anuncio del alcalde de Barcelona, Xavier Trias, de su intención de privatizar la gestión de las escuelas infantiles de la ciudad. Véase CIA (2011) y EUROPA PRESS (2011).

${ }^{4}$ Cabe destacar además que el Plan EEE no sólo se ha centrado en la economía considerada productiva, ignorando así el ámbito de la reproducción sino que además lo ha hecho en sectores económicos insostenibles como la construcción y que se caracterizan por estar fuertemente masculinizados. Según datos de la EPA en el tercer trimestre del 2008 la construcción ocupaba al 16\% de los hombres y únicamente al 1,9\% de las mujeres.

5 Independientemente de la divergencia de posturas existente en el seno de los feminismos ante medidas como el cheque-bebé o la misma existencia del Ministerio de Igualdad, divergencia que no es posible examinar aquí, su desaparición refuerza la tesis de una retirada del gobierno del PSOE de las políticas de apoyo a las familias y las mujeres.

6 Con un incremento de la carga total del trabajo nos referimos al aumento de horas de trabajo, tanto remunerado como no remunerado, de las mujeres. 
generalmente nos encontramos las mujeres; segundo, la evasión también por parte de los mercados de cualquier tipo de responsabilidad hacia el vacío o ausencia de cuidado que nuestra presencia en el mundo del empleo provoca y; tercero, el impacto de la crisis sistémica actual en nuestras dobles jornadas y, por ende, en la crisis de los cuidados.

A pesar del importante avance de las mujeres en el mundo del trabajo remunerado, en el estado español las mujeres constituimos únicamente el $43 \%$ de los contratos indefinidos y el $77 \%$ de las personas que trabajan a tiempo parcial. De todas las personas empleadas a media jornada, las mujeres sumamos el 97,07\% de las que alegan cuidado de niños o adultos y el $94,16 \%$ de las que presentan otras obligaciones familiares. Por otro lado, el $80 \%$ de las personas inactivas que no reciben ningún tipo de pensión son mujeres también. El salario medio de las mujeres ocupadas es un $22 \%$ menor al de los hombres y seguimos siendo víctimas de la segregación vertical y horizontal en el mercado laboral, así como de la discriminación de las mujeres embarazadas y de las mujeres con reducción de jornada por maternidad. Finalmente, las mujeres tenemos una mayor presencia que los hombres en la economía sumergida, con la ausencia de derechos laborales y sociales que ello comporta. Somos el $57,3 \%$ de personas receptoras de prestaciones no contributivas y únicamente el $37 \%$ de las contributivas. Cuando recibimos éstas últimas lo hacemos durante menos tiempo y en una cantidad un $15 \%$ menor a la de los hombres (véase Ezquerra, 2011b).

No nos contentamos con denunciar aquí, no obstante, la posición secundaria de las mujeres en el mercado laboral o la vulnerabilidad y precariedad que caracterizan nuestra presencia en él. Éstas encuentran su raíz y coartada en la persistencia de nuestra identificación social como responsables de la reproducción: lejos de ser entidades independientes, el ámbito llamado privado o de la familia y el ámbito llamado público se encuentran atravesados y vinculados por relaciones profundas y dialécticas. Cabe destacar en este sentido que las cifras laborales femeninas constituyen un síntoma no sólo de que el viejo consenso de género se había resquebrajado en menor medida de lo que podía parecer sino también de que el mercado laboral no ha asumido responsabilidad alguna por el vacío de presencia y de cuidado que la feminización del empleo de las últimas décadas ha generado. Los mercados no han creado un espacio real para la reproducción ni la han absorbido total o parcialmente sino que, en realidad, con la connivencia de los estados, han promovido la opresión de las mujeres en el ámbito de lo público y han dejado nuestra opresión privada lo más intacta posible. La lógica de la competitividad y el beneficio económico necesitó y permitió hace décadas la emancipación laboral de las mujeres, pero sólo de manera parcial. Lejos de caminar hacia prácticas de conciliación real, los mercados han resuelto las tensiones generadas por dicha emancipación parcial mediante la precarización y flexibilización del empleo femenino. Es precisamente en el frágil equilibrio resultante de la relación simbiótica entre nuestra entrada a medias al mundo del empleo y nuestra salida también a medias del hogar donde 
reside uno de los pilares fundamentales del capitalismo patriarcal y el caldo de cultivo para el estallido de la crisis de los cuidados en años recientes.

La crisis sistémica actual y su gestión política agudizan este escenario, ya que provocan una creciente informalización de los contratos laborales y resultan cómplices de un aumento de la violencia de género y el mobbing laboral contra las mujeres (véase Gálvez \& Torres, 2009). Más allá de sus efectos desastrosos sobre el conjunto de las clases populares, medidas pretendidamente anti-crisis como la Reforma Laboral y la Reforma de las Pensiones agudizan particularmente la precariedad laboral y vital de las mujeres. La Reforma Laboral eliminó la bonificación para la contratación de las mujeres, la cual no pasó a ser reemplazada por un ataque real a las causas estructurales de nuestra discriminación laboral. Además, mantuvo y fortaleció los incentivos a la contratación parcial, principal motor de la dualización de género del mercado de trabajo remunerado. Su introducción de mayor movilidad perjudica comparativamente a las mujeres por que solemos disponer de menor flexibilidad que los hombres. La Reforma de las Pensiones y su ampliación del período de cálculo de cotización, a su vez, agudiza la feminización de la pobreza ya que, a raíz de nuestra concentración en la economía informal, así como de la frecuente interrupción de nuestra vida laboral para cuidar de hijos y otras familiares, las mujeres encontraremos mayores dificultades a la hora de sumar la cotización exigida para obtener una pensión de jubilación digna (Ezquerra, 2011a).

La aceleración del crecimiento de las "tasas de actividad” femeninas desde que estalló la crisis mostrada en el Gráfico $1^{7}$, de este modo, no se traduce en una mayor autonomía ni en una cierta liberación de las mujeres respecto a las responsabilidades del cuidado. La destrucción masiva de empleo considerado masculino desde el tercer trimestre del 2008 ha provocado que muchas mujeres se incorporen al mercado laboral y que haya más familias que dependan del salario de la mujer, que en general suele ser el más bajo de los dos cónyuges ${ }^{8}$. Ello, no obstante, no se ha visto acompañado por una redistribución más equitativa de las responsabilidades domésticas y de cuidado en los hogares (Larrañaga, 2009). Si además tenemos en cuenta el aumento de la carga de trabajo doméstico sobre las mujeres como resultado de las estrategias para reducir gastos (Benería, 2003), así como de la disminución de recursos familiares para adquirir servicios remunerados de cuidado (Gálvez \& Torres, 2010), todo ello ha contribuido al aumento de la carga global de trabajo de las mujeres, al reforzamiento de nuestra doble jornada y a la diminución de nuestra calidad de vida y la del conjunto de nuestras familias. En definitiva, desde el punto de vista del mercado laboral y nuestra relación con él, la crisis sisté-

7 Mientras que en el año 2007 la “tasa de actividad” femenina fue del 48,94\%, en el 2008 fue de 50,46\%, en el 2009 de 51,57\%, en el 2010 de 52,27\% y en los tres primeros trimestres del 2011 de 52,91\%.

8 El incremento reciente en las "tasas de actividad" femenina se ha dado principalmente entre mujeres entre 25 y 54 años pero también entre las mayores de 54. 
mica también agrava la crisis de los cuidados y la carga sobre las espaldas de las mujeres.

En tercer lugar, como ya ha sido brevemente apuntado, la (insuficiente) respuesta de los hombres ante el silencioso estallido de la crisis de los cuidados ha contribuido también a su cierre reaccionario y agravamiento. Según los datos de la Encuesta de Empleo del Tiempo de los años 2009 y 2010 del Instituto Nacional de Estadística, aunque durante los últimos siete años la participación masculina en las tareas domésticas ha aumentado en casi cinco puntos, sigue existiendo una diferencia importante de participación en el trabajo no remunerado de 17 puntos porcentuales a cargo de las mujeres (74,7\% los hombres y 91,9\% las mujeres). Si bien durante los mismos años también se ha reducido en más de media hora la diferencia del tiempo medio dedicado al trabajo doméstico entre hombres y mujeres, el tiempo dedicado por las mujeres continúa superando al de los hombres en más de dos horas 9 . La persistencia de las diferencias se ha visto confirmada por estudios recientes de María Ángeles Durán (2011) y la OCDE (2011) sobre el trabajo no remunerado en la economía global.

Los recortes y privatizaciones de los servicios sociales públicos, los ataques a los derechos sociales y laborales, así como los impactos de todos ellos sobre las mujeres, no son meros efectos colaterales de la actual crisis sistémica y su gestión política, sino que constituyen una de sus principales estrategias en su huida hacia adelante. El concepto de Marx de "acumulación originaria"10 como proceso fundacional de la sociedad capitalista deviene clave en este punto. Según él, por acumulación originaria se entiende el conjunto de transformaciones que sirvieron de palanca para el nacimiento del sistema capitalista. Marx centró su análisis en la violenta expropiación de las tierras y de todos los medios de subsistencia del campesinado por parte de la emergente clase capitalista (Marx, 2007 [1867]: 200).

En los últimos años Silvia Federici y otras autoras han llevado a cabo una revisión del concepto marxiano desde una perspectiva feminista crítica (Federici \& Fortunati, 1987; Federici, 2010). Sus principales aportaciones residen en dos ejes. Por un lado, visibilizan a las mujeres y el trabajo reproductivo como víctimas fundamentales del proceso de acumulación originaria: el nacimiento del capitalismo no se erige únicamente sobre la proletarización de los hombres sino que es posible gracias a la ficticia separación de trabajo productivo y reproductivo, a la atribución de éstos a hombres y mujeres respectivamente y a la invisibilización y subordinación del segundo respecto al primero ${ }^{11}$. Es este proceso lo que en última instancia garantiza la "solvencia” del nuevo sistema.

\footnotetext{
9 Principales resultados de la Encuesta de Empleo del Tiempo 2009-2010 del INE disponibles en http://www.ine.es/prensa/np669.pdf (INSTITUTO NACIONAL DE ESTADÍSTICA, 2011)

10 También denominada acumulación primitiva.

11 Para un análisis en mayor profundidad de la naturaleza patriarcal, a la vez que etnocéntrica, de la acumulación originaria véase MIES (1998) [1986] y, entre otros, GALEANO (2003) [1971].
} 
Por otro lado, argumentan, lejos de ser un fenómeno exclusivamente fundacional, la acumulación originaria ha constituido un proceso universal en cada fase del desarrollo capitalista bajo la forma de múltiples estrategias relanzadas ante cada crisis del sistema cuyo objetivo ha sido abaratar el coste del trabajo remunerado y esconder la explotación de las mujeres y los sujetos coloniales ${ }^{12}$ (Federici, 2010: 31). Si en el contexto fundacional dichas estrategias consistieron en la pauperización por parte de la nueva clase dirigente de las mujeres, el despojo de su autonomía y su subordinación a la economía llamada productiva, entendemos que en el momento presente de crisis sistémica la acumulación originaria es actualizada por instituciones como el Banco Mundial, el Fondo Monetario Internacional y la Unión Europea mediante la imposición de programas de recortes, privatizaciones y austeridad (primero en el Sur global y en la actualidad en Europa). El principal objetivo y resultado de estos programas es el retorno al marco de los hogares (léase mujeres) de las responsabilidades de cuidado y reproducción (así como su externalización a los mercados) que los diferentes estados asumieron parcialmente tras la Segunda Guerra Mundial ${ }^{13}$.

De esta manera, el aumento de la carga total de trabajo de las mujeres como resultado de la crisis sistémica y de su gestión política, no constituye un mero (y pretendidamente inevitable) efecto colateral coyuntural sino que más bien responde a una estrategia político-económica consciente de las clases dirigentes de re-privatización de la reproducción en aras de la supervivencia de la economía considerada real. Cuando al capital le ha interesado y se lo ha podido permitir, sus ejecutores políticos no han dudado en cuestionar parcialmente los roles tradicionales de género. Sin embargo, en el momento en que sus contradicciones se ponen de manifiesto, dichos roles, que lejos de haberse extinguido permanecían vivos de manera más o menos latente, vuelven a cobrar fuerza como principal salvaguarda del orden económico y social. Si el estado puede externalizar sus responsabilidades de cuidado es porque cuenta con que las familias (léase mujeres) las asumirán (de nuevo $)^{14}$. La crisis sistémica, así, acelera y reafirma el cierre reaccionario, agravamiento y cronificación de la crisis de los cuidados y proporciona al estado la excusa perfecta para evadir todo tipo de responsabilidad respecto a la reproducción social.

En definitiva, las respuestas del estado, los mercados y los hombres contribuyen a que las mujeres continuemos siendo consideradas como las principales abastecedoras del cuidado y la reproducción (Ezquerra, 2010a). Ello se da de manera paralela a nuestra sobreexplotación específica en el mercado laboral y a la multiplica-

12 David Harvey (2004) ha trabajado también el concepto de acumulación primitiva en la forma particular de lo que él ha llamado desposesión (dispossession) como fundamental para entender las estrategias seguidas por el capital para salvarse de las crisis que él mismo genera.

13 Dicha devolución no es nueva sino que se inicia tras la crisis de los años 70 .

14 Celestino Corbacho, ministro de trabajo e inmigración durante el gobierno del PSOE, explicaba en una entrevista al diario Público el 18 de enero del 2010 la importancia de las familias en el estado español como cojín social. 
ción de nuestra doble presencia o, como matiza María Jesús Izquierdo (2003), cristaliza en una doble presencia-ausencia, la cual se ve agudizada con la crisis sistémica. Dicho esto, las preguntas que nos proponemos responder en el siguiente apartado son, en primer lugar ¿qué estrategias hemos seguidos las mujeres durante los últimos años para mitigar nuestra doble presencia-ausencia? En segundo lugar, ¿en qué marco político-social se han inscrito estas estrategias? Y en tercer lugar, ¿hasta qué punto la crisis sistémica actual ha alterado nuestras estrategias y/o los marcos en qué se desarrollan?

\section{EXTERNALIZACIÓN E INTERNACIONALIZACIÓN DE LA CRISIS DE LOS CUIDADOS}

En relación a las estrategias seguidas por las mujeres durante los últimos años para resolver o mitigar nuestra doble presencia-ausencia, cabe señalar que aquéllas que se lo han podido permitir han recurrido a la compra de una presencia y un cuidado mercantilizados realizados crecientemente por mujeres de origen inmigrante hasta hace bien poco. En la Tabla 1 se puede observar cómo mientras que en el año 1996 las personas de origen inmigrante (en su gran mayoría mujeres) constituían un 7\% del total de empleadas y empleados del hogar en el estado español, su presencia en el sector en 2009 sobrepasaba el 62\%:

Tabla 1. Evolución de empleados/as del hogar 1996-2009, miles de unidades y \%

\begin{tabular}{|c|c|c|c|c|c|c|c|c|c|c|c|}
\hline \multirow{2}{*}{ Año } & \multicolumn{2}{|c|}{ Autóctonas } & \multicolumn{2}{|c|}{ Inmigrantes } & \multirow{2}{*}{ Total } & \multirow{2}{*}{ Año } & \multicolumn{2}{|c|}{ Autóctonas } & \multicolumn{2}{|c|}{ Inmigrantes } & \multirow{2}{*}{ otal } \\
\hline & subtotal & & subtotal & & & & subtotal & & subtotal & & \\
\hline 1996 & 206 & 93,0 & 15,5 & 7,0 & 221,5 & 2003 & 229,9 & 56,0 & 180,4 & 44,0 & 410,3 \\
\hline 1997 & 218,3 & 92,6 & 17,3 & 7,4 & 235,7 & & 220 & 46,9 & 249,5 & 53,1 & 469,5 \\
\hline 1998 & 218,6 & 89,9 & 24,6 & 10,1 & 243,1 & 200 & 241,2 & 46,4 & 278,5 & 53,6 & 519,7 \\
\hline 1999 & 243,1 & 85,3 & 42 & 14,7 & 285,1 & 2006 & 228,2 & 41,3 & 324,6 & 58,7 & 552,8 \\
\hline 2000 & 240,4 & 81,2 & 55,7 & 18,8 & 296,1 & 200 & 250,7 & 42,9 & 333,2 & 57,1 & 583,9 \\
\hline 2001 & 239,9 & 74,7 & 81,1 & 25,3 & 321 & 201 & 229,7 & 42,1 & 316,2 & 57,9 & 545,8 \\
\hline 2002 & 219,7 & 64,5 & 121 & 35,5 & 340,7 & $2009 *$ & 191,8 & 37,4 & 320,7 & 62,6 & 512,5 \\
\hline
\end{tabular}

Elaboración propia a partir de datos del INE. Los datos se refieren al cuarto trimestre del año excepto en el caso de 2009, que se refieren al segundo trimestre. 
En lo que se refiere al ámbito político-social, el actual marco regulador del empleo en el hogar en el estado español, el Real Decreto (RD) 1424/1985, legaliza desde su nacimiento en el año 1985 cuestiones como la ausencia de contrato escrito, la presunción de temporalidad, el pago en especie, el despido libre barato y la incapacidad de los inspectores de trabajo de acceder a este ámbito de empleo. Además, su ambigüedad ha desembocado en numerosos vacíos legales que han permitido jornadas de más de doce horas sólo parcialmente retribuidas y el despido libre prácticamente gratuito. Todas estas vaguedades son justificadas con el argumento de que, a diferencia de otras actividades remuneradas, el empleo del hogar constituye una relación laboral “especial”. El estado español no es un caso aislado, y el trabajo doméstico no suele estar reconocido en ningún país como actividad laboral o trabajo "de verdad” (Martínez Veiga, 1995; López \& Toscani, 2006; Castelló, 2007; Ezquerra, 2008a; 2011c).

El RD 1424/1985, de esta manera, en gran medida viene cumpliendo la función de legalizar la desregulación e informalidad de un trabajo remunerado que suple las negligencias del estado, del mercado y de los hombres. Esta legalización contribuye a eximir al estado de su responsabilidad por el bienestar y el cuidado, la transfiere a unas trabajadoras condenadas a una precariedad perversamente regulada y hace que el repliegue de "lo público" resulte relativamente asequible a las familias que lo suplen mediante su externalización en el mercado.

Mientras que el RD 1424/1985 cristaliza las lamentables condiciones bajo las que se lleva a cabo esta actividad laboral, la Ley de Extranjería ha canalizado durante las últimas décadas la entrada de miles de mujeres inmigrantes al estado español ofreciéndoles como única oportunidad laboral y vital la realización de unas tareas respecto a las que el estado, los mercados y los hombres se lavan las manos (Mestre, 2001; Ezquerra, 2010a). La disponibilidad de un ejército de reserva de cuidadoras profanas inmigrantes es resultado a su vez del creciente éxodo de millones de mujeres de países del Sur global a raíz de la reestructuración económica impuesta desde el Norte y sus instituciones financieras desde la década de los ochenta. Basadas en principios idénticos a los que rigen el actual plan de austeridad europeo, la aplicación de estas políticas en el Sur global ha desembocado en la eliminación de la agricultura de subsistencia, en una mayor concentración de los medios de producción y la riqueza, en elevadas tasas de paro y subocupación y en una asfixia del sector público. Estos procesos han golpeado de manera particularmente severa a las mujeres, ya que tradicionalmente éstas han tenido una importante presencia en la pequeña agricultura y también en sectores públicos como la enseñanza y la sanidad. Además, a raíz del recorte de presupuestos sociales, como aquí, son las principales substitutas del estado a la hora de cuidar a los más vulnerables.

Ante la imposibilidad de mantener a sus propias familias, muchas de ellas han emigrado a lugares como el estado español para cuidar a los seres queridos de miles de familias mientras que dejan a las suyas en manos de maridos, hermanas, madres o amigas. De esta manera, el cierre reaccionario de la crisis de los cuidados 
en el estado español ha sido posible gracias a la importación de centenares de millares de cuidadoras como resultado de la crisis de reproducción social en el Sur global. Su diáspora no sólo ha contribuido a enmascarar la irreconciliable contradicción existente entre el capitalismo patriarcal y el mantenimiento de la vida en el Norte sino que además, ante los procesos de reestructuración económica en el Norte y en el Sur, estas mujeres por un lado han subvencionado a nuestros gobiernos cargando sobre sus espaldas, y en condiciones de extrema precariedad, las tareas de la reproducción de la vida y, por el otro, han mantenido "a flote” a los gobiernos y las economías de origen mediante el envío de remesas masivas que en muchos lugares han superado con creces cualquier otra fuente de divisas y han devenido fundamentales para la supervivencia de una parte importante de la población. Irónicamente, huyen de unos estados negligentes para acabar subvencionando a otros que no lo son menos.

En el marco de una creciente división internacional del trabajo, los derechos e incluso los afectos, mientras que estas mujeres han cubierto durante años nuestros vacíos de presencia y cuidados, sus hijos e hijas se han criado lejos de ellas. Una vez alcanzada la vida adulta, muchos y muchas de ellos han seguido cruzando fronteras para venir a substituir a sus madres y servir a otros y a otras, dejando a su vez a sus propias familias atrás (Parreñas, 2001; 2005). De esta manera, la "cadena transnacional del cuidado" ha garantizado durante años la pervivencia del capitalismo patriarcal a la vez que ha invisibilizado su naturaleza excluyente.

La crisis de los cuidados no ha sido resuelta, de esta manera, mediante una generalizada y equitativa implicación de los hombres en el trabajo del cuidado, ni un fortalecimiento de las responsabilidades reproductivas del estado ni, por descontado, una feministización del mercado laboral. La entrada de cientos de miles de mujeres inmigrantes durante los últimos años en los hogares españoles como mano de obra reproductiva enmascara la opresión patriarcal de las mujeres autóctonas, ya que a menudo éstas, en lugar de optar por cuestionar activamente los roles de género en el seno de la familia, han delegado parte del suyo en mujeres en clara posición de subordinación por su origen nacional, posición socioeconómica y situación administrativa. Este deslizamiento de la división sexual del trabajo ha descansado sobre la explotación laboral de las "substitutas” y su discriminación en un mercado laboral profundamente segmentado. Las contradicciones visibilizadas por la crisis de los cuidados han sido así gestionadas hasta hace bien poco mediante una externalización e internacionalización de la solución, las cuales, además de eximir al estado, al mercado y a los hombres de gran parte de su responsabilidad reproductiva, han dado entrada a nuevos ejes de desigualdad y han acentuado los previamente existentes (Ezquerra, 2010a).

Estos procesos, sin embargo, se han visto parcialmente interrumpidos por la crisis sistémica desde finales del año 2008. En ese momento, el entonces ministro de trabajo e inmigración, Celestino Corbacho, anunciaba el fin de las contrataciones en origen de personas extranjeras y, ante las crecientes tasas de paro, la prioriza- 
ción de su gobierno de las personas autóctonas desempleadas. ${ }^{15}$ Cabe diferenciar los impactos de dicho giro sobre la población de origen inmigrante por un lado y la población autóctona por el otro.

La primera ha visto desde entonces su acceso físico al estado español severamente limitado. Dicha limitación se refleja en el reciente declive de nuevos residentes extracomunitarios y de la cantidad de empleos de difícil cobertura mediante los cuales las personas extranjeras han accedido durante los últimos años a un permiso de empleo y, por ende, también de residencia ${ }^{16}$. La crisis sistémica también ha disparado de manera desproporcionada las tasas de desempleo formalmente reconocido de la población inmigrante, las cuales se sitúan en la actualidad en el $27,15 \%$ para las mujeres y cerca del $34 \%$ para los hombres ${ }^{17}$. En último lugar, las personas inmigrantes están sufriendo desde el estallido de la crisis sistémica su utilización como chivos expiatorios de la crisis por diversos discursos y prácticas políticas. El ascenso del xenófobo Josep Anglada en Catalunya durante los últimos años, la expansión de la "polémica del burka" o, entre otras cuestiones, la exclusión de las personas inmigrantes de algunos servicios y derechos sociales por parte de administraciones como la catalana, son síntomas y dramáticas consecuencias de ello.

La pérdida de poder adquisitivo y creciente pauperización de una parte importante de la población autóctona, por otro lado, hace que las familias dispongan de menos recursos, como se ha expuesto anteriormente, para acceder al trabajo reproductivo mercantilizado y que realicen en su seno tareas por las que hasta hace poco pagaban dinero. Este crecimiento de lo que Amaia Pérez Orozco, inspirada en Natalia Ribas-Mateos, denomina en este mismo volumen la economía de retales, aumenta, tal y como venimos mostrando, la carga total de trabajo de las mujeres y potencia la exclusión laboral de las que son inmigrantes. En definitiva, con la crisis sistémica no sólo se agrava y cronifica la crisis de los cuidados sino que también se visibiliza la fragilidad de su supuesta solución: la importación y contratación precaria de mujeres como parche y como último (y a menudo único) cojín para cuidar, cuidarse o ser cuidados/as. La (supuesta) relativa tolerancia del capital en tiempos

15 LARREA, Ainhoa (2008) "Corbacho quiere restringir los contratos en origen de inmigrantes" (Público, 3/9/2008) http://www.publico.es . Para un análisis de las implicaciones del giro en la política de extranjería véase EZQUERRA, Sandra (2008b) "Més enllà de les declaracions de Corbacho: immigració i neoliberalisme a l'Estat español”, disponible en http://www.revoltaglobal.cat/article1743.html

16 Según los datos del INE, mientras que en el año 2008 hubo un total de 790.205 contrataciones a personas extranjeras en el 2009 esta cifra se redujo a poco más de la mitad. A su vez, mientras que el Catálogo Trimestral de Ocupaciones de Difícil Cobertura, principal herramienta para la ordenación de la entrada de mano de obra no comunitaria, contenía en el año 2005 más de 40 páginas llenas de tipos de empleos a los que las personas inmigrantes podían aspirar, entre ellas el empleo del hogar y múltiples empleos relacionados con el cuidado, a inicios del 2011 apenas incluía unos cuantos empleos, entre los que no figuraban ni el empleo del hogar ni la atención o cuidado. El Catálogo Trimestral de Ocupaciones de Difícil Cobertura es publicado trimestralmente en el Boletín Oficial del Estado•

17 Ante la falta de datos, podemos lanzar la hipótesis que dada la concentración de las personas inmigrantes en la economía informal (hostelería, empleo doméstico, prostitución, etc.), estas cifras en realidad son mucho más altas. 
de bonanza hacia los (lentos) avances ${ }^{18}$ en los derechos de mujeres autóctonas e inmigrantes se disipa con la llegada de las vacas flacas.

\section{CONCLUSIONES}

En el presente texto se ha realizado un esfuerzo para analizar algunos de los factores principales generadores de la crisis de los cuidados y se han examinado algunas respuestas políticas, económicas y sociales a ellas. Se ha mostrado que principalmente el estado y los mercados han evadido abordar las premisas y desigualdades que yacen tras la crisis de los cuidados y han intentado mitigarla promoviendo su externalización e internacionalización.

Por otro lado, la gestión neoliberal de la crisis sistémica manifestada hace tres años ha agravado y cronificado la crisis de los cuidados y ha mostrado la fragilidad de las respuestas ante ella. Las políticas de subordinación a los mercados que los gobiernos vienen adoptando constituyen, a su vez, una actualización de los procesos de acumulación originaria mediante la devolución a las mujeres en los hogares de una parte importante de las responsabilidades del cuidado y la reproducción previamente asumidas por el estado, así como su externalización al sector privado, como parte de una estrategia de re-privatización de la reproducción en aras de la supervivencia y enriquecimiento de los mercados. Dicha respuesta no sólo define cada vez con más claridad el rumbo de las políticas de los gobiernos en los próximos años sino que sobre todo nos recuerda de nuevo el íntimo, aunque invisibilizado, vínculo existente entre la economía considerada productiva y la reproductiva, así como la centralidad, de nuevo invisibilizada, de los cuidados y la reproducción en lo que muchos se empeñan en seguir llamando economía real. Reivindicarla será una de las principales tareas de los feminismos del siglo XXI.

\section{BIBLIOGRAFÍA}

BENERÍA, Lourdes (2003a) "Economic Rationality and Globalization: A Feminist Perspective", en Ferber, M. \& Nelson, J. Feminist Economics Today. Beyond Economic Man, Chicago, The University of Chicago Press

BENERÍA, Lourdes (2003b) Gender, Development, and Globalization. Economics as If People Mattered, Nueva York, Routledge.

CASTELLÓ, Laia (2007) Les formes de gestió del treball domèstic assalariat a l'Estat

Espanyol. Departament de Sociologia de la Universitat Autònoma de Barcelona.

17 La afirmación de que los derechos de las mujeres inmigrantes se han incrementado durante los últimos años es como mínimo dudosa y la autora es consciente de ello. Se dan dinámicas posiblemente contradictorias provenientes del ámbito institucional, social, económico, cultural, etc. Analizar dichas contradicciones requeriría un espacio del que no disponemos aquí pero se considera que en las esferas laborales, sociales, e incluso culturales, ha habido tímidas y, como se argumenta en el texto frágiles, mejoras. 
CIA, Blanca (2011) "Xavier Trias entierra el modelo de guarderías públicas en Barcelona” (El País, 16/9/2011) http://www.elpais.com

DEL RÍO, Sira (2004) "La crisis de los cuidados: precariedad a flor de piel". Documento de trabajo. CGT-Comisión Confederal contra la Precariedad

DURÁN, María Ángeles (2011) El trabajo no remunerado en la economía global, Madrid, Fundación BBVA

EZQUERRA, Sandra (2008a) The Regulation of the South-North Transfer of Reproductive Labor: Filipino Women in Spain and the United States, University of Oregon. Eugene (Estados Unidos) Eugene, OR: University of Oregon. Tesis doctoral disponible en http://scholarsbank.uoregon.edu/jspui/handle/1794/9017

EZQUERRA, Sandra (2008b) "Més enllà de les declaracions de Corbacho: immigració i neoliberalisme a l'Estat español”, disponible en http://www.revoltaglobal.cat/article1743.html

EZQUERRA, Sandra (2010a). "La crisis de los cuidados: orígenes, falsas soluciones y posibles oportunidades”. Viento Sur, núm. 108

EZQUERRA, Sandra (2010b) “A propósito de las crisis ¿qué pasa con la de los cuidados?” Diagonal, núm. 121. 4/3/2010

EZQUERRA, Sandra (2011a) "Crisis desiguales: miradas feministas a los efectos de la crisis”, Viento Sur, núm. 114, pp. 91-98

EZQUERRA, Sandra (2011b) "Women's Crises: Spanish economic recession and political responses from a feminist perspective” en Onaran \& Leplat (eds.) Capitalism and crisis, Londres, International Institute for Research and Education

EZQUERRA, Sandra (2011c) "Hogar privado y responsabilidad pública: un recorrido histórico por la regulación del trabajo doméstico en el Estado español”, en TERRADAS (coord.) Antropología jurídica de la responsabilidad, Santiago de Compostela, Andavira Editora

EUROPA PRESS (2011) "Trias desecha el modelo de guarderías de Hereu y descarta construir más centros públicos” (El Periódico, 15/9/2911) http://www.elperiodico.com

FEDERICI, Silvia \& FORTUNATI, Leopoldina (1987) Il Grande Calibano. Storia del corpa sociale ribelle nella prima fase del capitale, Milán, Franco Angeli Editore

FEDERICI, Silvia. 2010. Calibán y la bruja. Mujeres, cuerpo y acumulación originaria. Madrid: Traficantes de Sueños

FERBER, Marianne. \& NELSON, Julie (2003) Feminist Economics Today. Beyond Economic Man, Chicago, The University of Chicago Press

GALEANO, Eduardo (2003) [1971] Las venas abiertas de América Latina, Siglo XXI, Madrid

GÁLVEZ, Lina \& TORRES, Juan (2009) "La crisis económica y sus alternativas: una perspectiva de género", "Feminismo ante la crisis”, Fórum de Política Feminista, pp. 15-30, http://www.forumpoliticafeminista.org/fotos/files/1.pdf

GÁLVEZ, Lina \& TORRES, Juan (2010) Desiguales. Mujeres y hombres en la crisis financiera. Barcelona: Icaria

GOBIERNO DE ESPAÑA (2008) Plan español para el estímulo de la economía y el empleo.

Disponible

en http://www.meh.es/Documentacion/Publico/PortalVarios/Gesti\%C3\%B3n\%20del\% 20Portal/Plan\%20estimulo\%20econom\%C3\%ADa\%20y\%20empleo.pdf 
HARVEY, David (2004) “The 'New’ Imperialism: Accumulation by Dispossession”, Socialist Register, vol. 40, http://socialistregister.com/

HERNANDO, Ana (2006) "Una crítica feminista a la Ley de Dependencia”. Viento Sur, núm. 88, pp. 89-95

INSTITUTO NACIONAL DE ESTADÍSTICA (2011) Encuesta de Empleo del Tiempo 2009-2010, http://www.ine.es/prensa/np669.pdf

IZQUIERDO, María Jesús (2003) “Del sexismo y la mercantilización del cuidado a su socialización: Hacia una política democrática del cuidado”, en Congreso Internacional SARE Cuidar cuesta: costes y beneficios del cuidado, Donostia, Emakunde-Instituto Vasco de la Mujer y Comunidad Europea/Foro Social Europeo

LARRAÑAGA, Mertxe (2009) “Mujeres, tiempos, crisis: Combinaciones variadas”. Revista de Economía Crítica, núm. 8, pp. 113-120

LARREA, Ainhoa (2008) "Corbacho quiere restringir los contratos en origen de inmigrantes” (Público, 3/9/2008) http://www.publico.es

LÓPEZ, Juan \& TOSCANI, Daniel (2006) Los trabajadores al servicio del hogar familiar. Aspectos laborales y de seguridad social. Propuestas de reforma, Albacete, Editorial Bomarzo.

MARCHAND, Marianne \& RUNYAN, Anne (2000) Gender and Global Restructuring. Sightings, Sites and Resistances, Londres, Routledge

MARX, Karl (2007) [1867] El Capital, Madrid, Akal. Libro I, Tomo III.

MESTRE, Ruth (2001) Feminisme, dret i immigració: una crítica a la llei d'estrangeria, Valencia, Universidad de Valencia. Tesis doctoral

MIES, Maria (1998) [1886] Patriarchy \& Accumulation on a World Scale. Women in the International Division of Labour, Zed Books, Londres

NAVARRO, Vicenç; TORRES, Juan; GARZÓN, Alberto (2011) Hay alternativas. Propuestas para crear empleo y bienestar social en España, Madrid, Sequitur

OCDE (2011) Society at a Glance 2011 - OECD Social Indicators, http://www.oecd.org/els/social/indicators/SAG

OTXOA, Isabel (2009) “Anticapitalismo: algunas razones desde el feminismo”. Viento Sur, núm. 104, pp. 66-73

PARREÑAS, Rhacel (2001) Servants of globalization. Women, migration and domestic work, Standford, Standford University Press.

PARREÑNA, Rhacel (2005) Children of global migration. Transnational families and gendered woes, Standford, Standford University Press.

PÉREZ OROZCO, Amaia (2006) “Amenaza tormenta: la crisis de los cuidados y la reorganización del sistema económico”. Revista de Economía Crítica, núm. 5, pp. 7-37

RICO, María Nieves (2009) “Crisis de cuidado y políticas públicas. El momento es ahora”. Ponencia presentada en el Seminario Regional Las familias latinoamericanas interrogadas. Hacia la articulación del diagnóstico, la legislación y las políticas. Santiago de Chile, 29 y 30 de octubre de 2009.

SAAD-IMSERSO (2011) Estadísticas del sistema para la autonomía y la atención a la dependencia. $\quad$ http://www.imsersodependencia.csic.es/estadisticas/saad/saad2011.html

SALZINGER, Leslie (2003) Genders in Production, Berkeley, UC Press

SETIÉN, María Luisa \& ACOSTA, Elaine (2010) "La gestión de la crisis de los cuidados y su relación con la feminización de las migraciones. Análisis comparativo de los modelos de España y Chile”, Alternativas, núm. 17, pp. 63-81 